\title{
U so de endoprótesis en el tratamiento de lesiones no oclusivas del territorio iliaco
}

\author{
U so de endoprótese no tratamento de lesões não-oclus vas do território ilíaco
}

\author{
Renato M ertens, Albrecht Krämer, Francisco Valdés, Michel Bergoeing, Leopoldo M ariné, \\ Rodrigo Sagües, Ricardo O Iguín, Juan C ruz, M agaly Valdebenito, Jeanette Vergara*
}

\section{Resumen}

O bjetivo: La cirugía endovascular se ha establecido como una opción eficaz en el tratamiento de la enfermedad oclusiva ateroesclerótica de las arterias iliacas. Sin embargo, el uso de estos procedimientos para tratar otro tipo de lesiones aún no ha sido bien estudiado. N uestro objetivo es analizar indicaciones y resultados del uso de endoprótesis en lesiones iliacas no oclusivas.

M aterial y métodos: Revisamos retrospectivamente los registros de 14 pacientes consecutivos, todos hombres, 61,6 años de edad en promedio (rango: 25-80) tratados por vía endovascular entre 2001 y 2006 por lesiones iliacas no oclusivas. El estudio pre y postoperatorio incluyó tomografía computada. El procedimiento se efectuó en quirófano, utilizando un angiógrafo digital. Se usó acceso femoral insertando endoprótesis tubulares.

Resultados: En 11 pacientes se asoció embolización de arteria hipogástrica ipsilateral. Las patologías tratadas fueron: ocho aneurismas ateroescleróticos, 3 disecciones, 2 lesiones traumáticas y un pseudoaneurisma anastomótico. La co-morbilidad más frecuente fue la hipertensión en $43 \%$ de los casos. No hubo mortalidad operatoria. Un paciente presentó un pseudoaneurisma femoral tratado con compresión. Un paciente tratado por fístula arteriovenosa traumática a nivel iliaco resuelve su insuficiencia cardiaca, con persistencia asintomática de mínimo flujo. La estadía postoperatoria fue de 3 días (mediana). N o se presentaron otras endofugas iniciales o tardías. D urante un seguimiento promedio de 20,5 meses (rango 1 a 49), un paciente fallece por cáncer y ninguno ha requerido procedimientos complementarios.

Conclusión: El tratamiento endovascular de lesiones iliacas mediante endoprótesis es seguro y permite un resultado durable en el manejo de un amplio espectro de patologías.

Palabras clave: Arteria ilíaca, endoprótesis, endovascular, aneurisma.

\section{Resumo}

O bjetivo: A cirurgia endovascular se estabeleceu como opção eficaz no tratamento da doença oclusiva aterosclerótica das artérias ilíacas. Entretanto, o uso desses procedimentos para tratar outros tipos de lesão ainda não foi bem estudado. N osso objetivo é analisar indicações e resultados do uso de endoprótese em lesões ilíacas não-oclusivas.

M aterial e métodos: Revisamos retrospectivamente os registros de 14 pacientes consecutivos, todos homens, com idademédia de 61,6 anos (faixa: 25-80 anos), tratados por via endovascular entre $2001 \mathrm{e}$ 2006 por lesões ilíacas não-oclusivas. 0 estudo pré e pós-operatório incluiu tomografia computadorizada. 0 procedimento foi realizado em bloco cirúrgico, utilizando um angiógrafo digital. Foi utilizado acesso femoral, com a inserção de endopróteses tubulares.

Resultados: Em 11 pacientes, houve embolização de artéria hipogástrica ipsilateral associada. As patologias tratadas foram: oito aneurismas ateroscleróticos, 3 dissecções, 2 lesões traumáticas e um pseudo-aneurisma anastomótico. A comorbidade mais freqüente foi hipertensão, em $43 \%$ dos casos. N ão houve mortalidade operatória. U m paciente apresentou um pseudo-aneurisma femoral tratado com compressão. U m paciente tratado por fístula arteriovenosa traumática em nível ilíaco teve sua insuficiência cardíaca resolvida, com persistência assintomática de mínimo fluxo. A estada pós-operatória foi de 3 dias (média). N ão se apresentaram outras endofugas inciais ou tardias. D urante um acompanhamento médio de 20,5 meses (faixa de 1 a 49 meses), um paciente morreu de câncer e nenhum requereu procedimentos complementares.

C onclusão: 0 tratamento endovascular de lesões ilíacas através de endoprótese é seguro e permite um resultado durável no manejo de um amplo espectro de patologias.

Palavras-chave: Artéria ilíaca, endoprótese, endovascular, aneurisma.

\footnotetext{
* Departamento de Enfermedades Cardiovasculares y División de Cirugía, Facultad de Medicina, Pontificia Universidad Católica de Chile, Santiago, Chile.

Artículo sometido el 04.04.06, aceptado el 12.06.06.
} 


\section{Introducción}

La cirugía endovascular, en comparación con la cirugía abierta convencional, reduce la magnitud de los procedimientos para tratar patologías vasculares, diminuyendo así la morbilidad y eventualmentela mortalidad. Además, permite una recuperación más expedita del paciente con una reinserción familiar y laboral precoz. En 1997, iniciamos un protocolo de tratamiento de enfermedades vasculares mediante el implante de endoprótesis, reportando nuestros resultados en numerosas patologías tanto aórticas ${ }^{1-4}$ como en otros territorios $^{5}$.

En la enfermedad oclusiva ateroesclerótica aortoiliaca, la terapia endovascular representada por la angioplastía percutánea y la inserción destents, se ha establecido como una opción globalmente aceptada por lo poco invasiva y sus buenos resultados a largo plazo ${ }^{6}$.

El uso de estas técnicas para el tratamiento de patologías no oclusivas en este territorio se ha concentrado en el tratamiento del aneurisma iliaco y sólo en forma ocasional se ha descrito su uso en otro tipo de lesiones. N uestro objetivo es analizar las indicaciones y resultados en el tratamiento de lesiones iliacas no oclusivas, en general mediante endoprótesis.

\section{Pacientes y método}

Revisamos en forma retrospectiva los registros de todos los pacientes tratados consecutivamente por vía endovascular por enfermedad del territorio ilíaco entre julio de 2001 y febrero de 2006.

D eun total de 124 procedimientos endovasculares iliacos en ese periodo, se identifican 14 realizados en el mismo número de pacientes por enfermedad no oclusiva $(11,3 \%)$, todos de sexo masculino, con una edad promedio de 61,6 años (rango: 25-80). Las patologías asociadas se encuentran descritas en la T abla 1.

T abla 1 - Patología asociada en 14 pacientes

\begin{tabular}{lc}
\hline Co-morbilidades & $\mathbf{n}(\%)$ \\
\hline H ipertensión arterial & $6(42,9)$ \\
Enfermedad coronaria & $3(21,4)$ \\
D islipidemia & $5(35,7)$ \\
Aneurisma aórtico operado & $5(35,7)$ \\
H emodiálisis & $2(14,3)$ \\
Insuficiencia cardiaca & $2(14,3)$ \\
Shock hipovolémico & $1(7,1)$ \\
\hline
\end{tabular}

El estudio preoperatorio incluyó tomografía computada helicoidal en fase arterial de rutina y angiografía convencional sólo selectivamente. El procedimiento se efectuó en el quirófano, utilizando un angiógrafo digital G eneral Electric/O EC ${ }^{\circledR} 9800$ (EEUU ) para la generación de imágenes y control del despliegue del dispositivo en el sitio anatómico preciso. Se usó acceso femoral para insertar la endoprótesis en todos los pacientes. En caso necesario, se asoció embolización dela arteriailiacainternaipsilateral mediante acceso femoral contralateral y el despliegue de coils espirales de 0,035 pulgadas y entre 5 y $10 \mathrm{~mm}$ de diámetro $\left(\operatorname{Cook}^{\circledR}\right.$, EEU U ). EI objetivo de la embolización es ocluir dicho vaso, generar trombosis y evitar flujo retrógrado hacia la arteria iliaca común (Figuras 1 y 2) o con el fin de excluir en forma completa un aneurisma localizado en la arteria iliaca interna, ocluyendo en forma individual todas sus ramas.

El seguimiento serealizó en formaclínica y mediante tomografía computada para evaluar morfológicamente la reparación. Endofuga fue definida como la

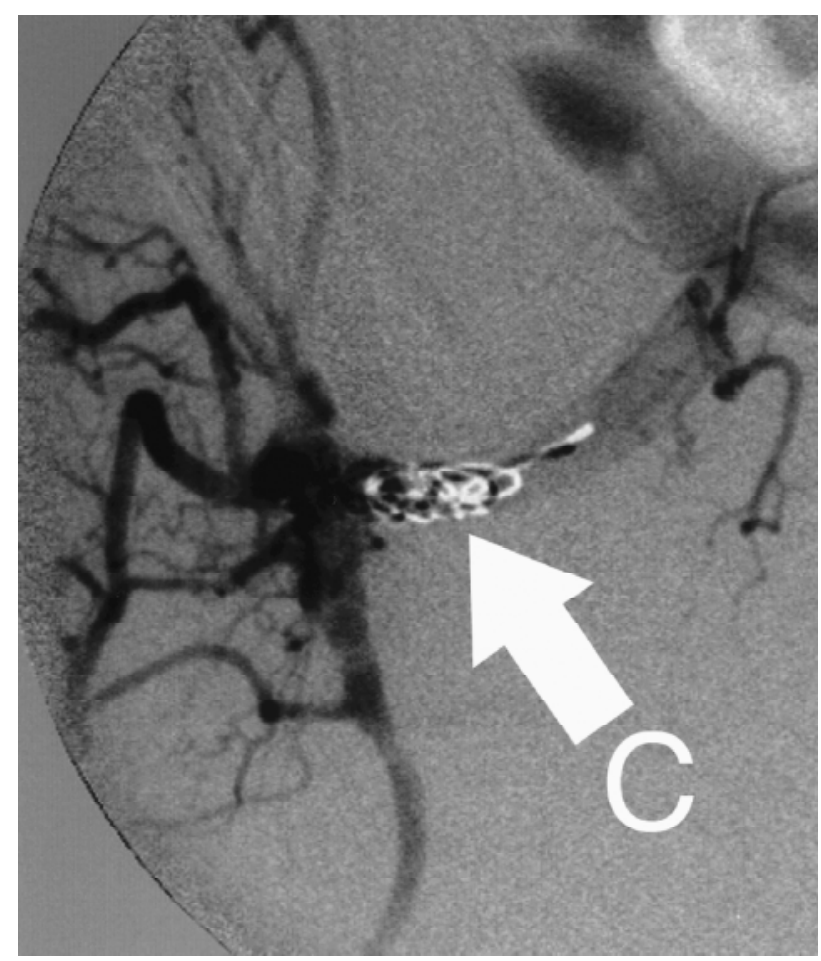

Figura 1 - I magen angiográfica durante embolización de la arteriahipogástrica, $\mathbf{C}$ muestralos coilsinstalados en el tronco del vaso previo al nacimiento de sus primeras ramas 
persistencia de flujo hacia la lesión tratada, ya sea directamente desde la circulación (tipo I) o en forma retrógrada a través de colaterales (tipo II). Se consideró como mortalidad operatoria aquella ocurrida intrahospital aria 0 antes de 30 días deefectuada la intervención.

\section{Resultados}

En los 14 pacientes, se completó exitosamente el procedimiento programado. Las patologías tratadas fueron diversas ( $T$ abla 2 ), destacando los aneurismas de arteria iliaca común (Figura 3) e interna (Figura 4), todos ellos mayores de $3,5 \mathrm{~cm}$ de diámetro. D os pacientes correspondían a disección ilíaca aislada con dilatación aneurismática crónica.

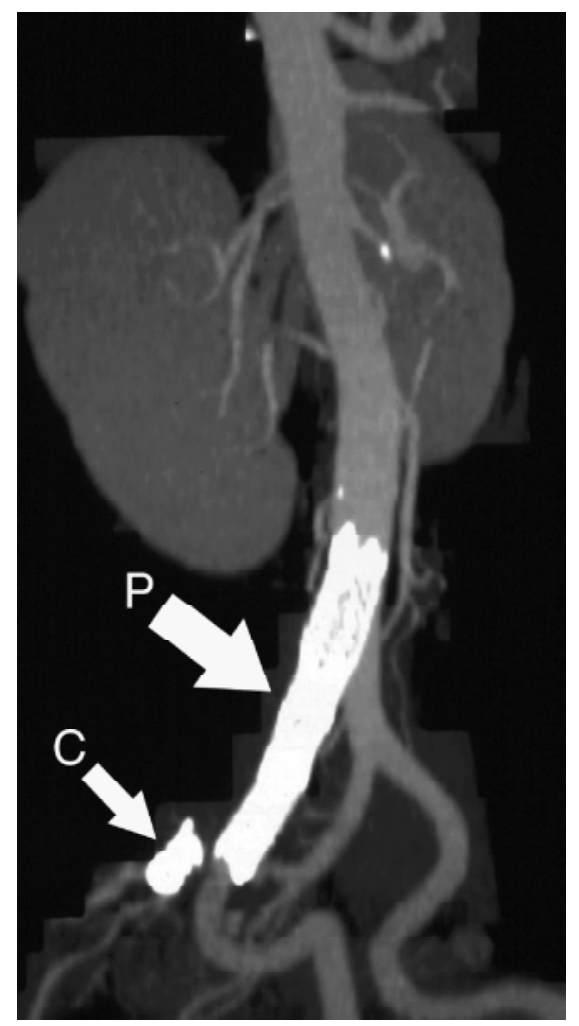

Figura 2 - Reconstrucción bidimensional de tomografía computada en proyección oblicua, muestrala endoprótesis (P) queseextiendeentrela arteriailiaca común ylaexterna. $\mathbf{C}$ muestraloscoils en la arteria hipogástrica evitando el flujo retrógrado hacia la lesión tratada
Tabla 2 - N aturaleza de la lesión

\begin{tabular}{lc}
\hline Tipo de lesión & $\mathbf{n}$ \\
\hline Aneurisma iliaco común aislado & 5 \\
Aneurisma hipogástrico aislado & 3 \\
Disección iliaca aislada espontánea & 3 \\
Trauma & 1 (fístula A-V) \\
& 1 (ruptura) \\
Pseudoaneurisma anastomótico & 1 \\
\hline
\end{tabular}

O nce pacientes eran asintomáticos y fueron tratados en forma electiva para evitar su complicación por ruptura. T res pacientes presentaban síntomas derivados de sus lesiones. Un paciente fue intervenido en forma urgente al presentarse con shock hipovolémico por ruptura iliaca, otro se presentó con insuficiencia cardiaca de alto débito por una fístula arterio venosa, ambos casos posterior a cirugía ortopédica de columna y finalmente un caso de disección iliaca común espontánea, con dilatación aguda del vaso y dolor perineal intratable (Figura 5).

La anestesia utilizada fue peridural en 3 pacientes, local más sedación en 7 y general en 4 casos, incluidos los 3 pacientes sintomáticos y otro por preferencia individual. La vía de acceso más frecuente fue la denudación femoral en 12 pacientes, por el al to diámetro del sistema de entrega de la endoprótesis (mayor a 11 french) que impide un uso percutáneo seguro. En dos casos se realizó en forma percutánea utilizando un sistema de cierre femoral (Perclose ${ }^{\circledR}$, Abott M edical, EEU U ). Los dispositivos utilizados se encuentran descritos en la T abla 3.

Tabla 3 - D ispositivos utilizados (extensión o rama iliaca son parte de dispositivos diseñados para el tratamiento de aneurismas de aorta abdominal)

\begin{tabular}{|c|c|}
\hline D ispositivo & n (\%) \\
\hline Extensión iliaca Zenith ${ }^{\circledR}$ (Cook, EEUU) & $8(57,2)$ \\
\hline W allgraft ${ }^{\circledast}$ (Boston Scientific, EEUU ) & $3(21,4)$ \\
\hline Extensión iliaca Talent ${ }^{\circledR}$ (M edtronic, EEUU ) & $2(14,3)$ \\
\hline Rama iliaca Excluder ${ }^{\circledR}$ (Gore, EEUU ) & $1(7,1)$ \\
\hline
\end{tabular}


En 11 pacientes se asoció embolización simultánea delaarteria hipogástrica; en 8, para obtener unazona de apoyo distal en la arteria iliaca externa, al comprometer la lesión la zona de la bifurcación iliaca; y en tres casos, fue parte integral del tratamiento de aneurismas de arteria iliaca interna. Sólo dos pacientes $(14,3 \%$ ) requirieron detransfusión. La mediana deestadía postoperatoria fue de 3 días (rango 1-12 días).

Dos pacientes presentaron complicaciones $(14,3 \%)$, uno presentó un pseudoaneurisma femoral post punción detectado una semana después del al ta, fue tratado en forma inmediata con compresión bajo ecografía y el otro una trombosis venosa profunda
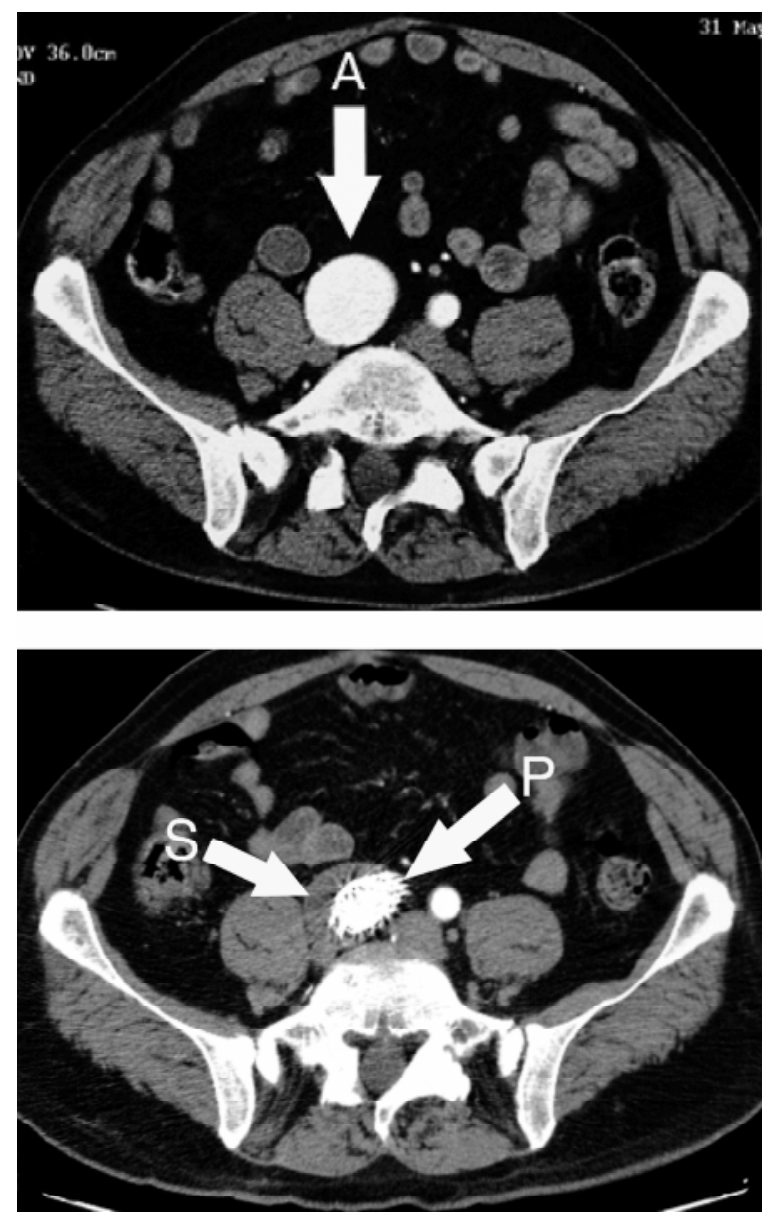

Figura 3 - Corte axial de tomografía computada que demuestra un gran aneurisma iliaco común derecho (A). M uestra la lesión tratada con endoprótesis $(\mathbf{P})$ generando trombosis del saco aneurismático (S) y disminución del diámetro de la lesión detectada en la misma hospitalización y tratada mediante la inserción de un filtro de vena cava inferior, al tener el paciente contraindicación de anticoagulación. No se presentaron otras complicaciones. No hubo mortalidad operatoria.

U n paciente tratado por fístula arteriovenosa iliaca post traumática presenta resolución del cuadro deinsuficiencia cardiaca de alto débito que motivó su tratamiento. En la tomografía computada de control previa al alta se demuestra persistencia asintomática de flujo residual mínimo a través de la fístula, constituyendo una endofuga de tipo I (7,1\%). D ebido a su magnitud y ausencia de síntomas se decidió manejo conservador. $\mathrm{N}$ o se presentaron otras endofugas iniciales.

El seguimiento promedio fue de 20,5 meses (mediana 23 meses, rango 1 a 49 meses, Tabla 4). Al momento del cierre, dos de los 11 pacientes tratados con embolización hipogástrica presentan claudicación glútea $(18,2 \%)$. En uno de ellos se presenta a corta distancia, limitando parcialmente sus actividades. U n paciente fallece de cáncer a los 21 meses. $N$ ingún

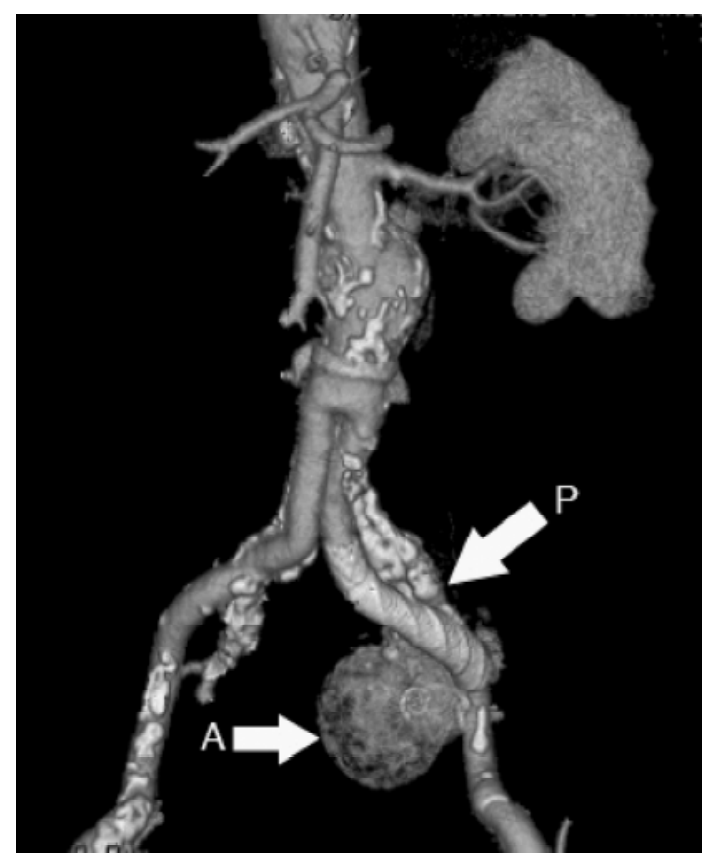

Figura 4 - Reconstrucción tridimensional detomografíacomputada quemuestra una endoprótesis $(\mathbf{P})$ entre iliaca común y externa izquierdascon exclusión deun gran aneurisma hipogástrico (A), que se observa trombosado 
paciente ha requerido procedimientos complementarios, presentado otras complicaciones derivadas de la intervención 0 aparición tardía de endofugas.

\section{Discusión}

La descripción de la utilización de endoprótesis en el tratamiento del aneurisma de aorta abdominal por
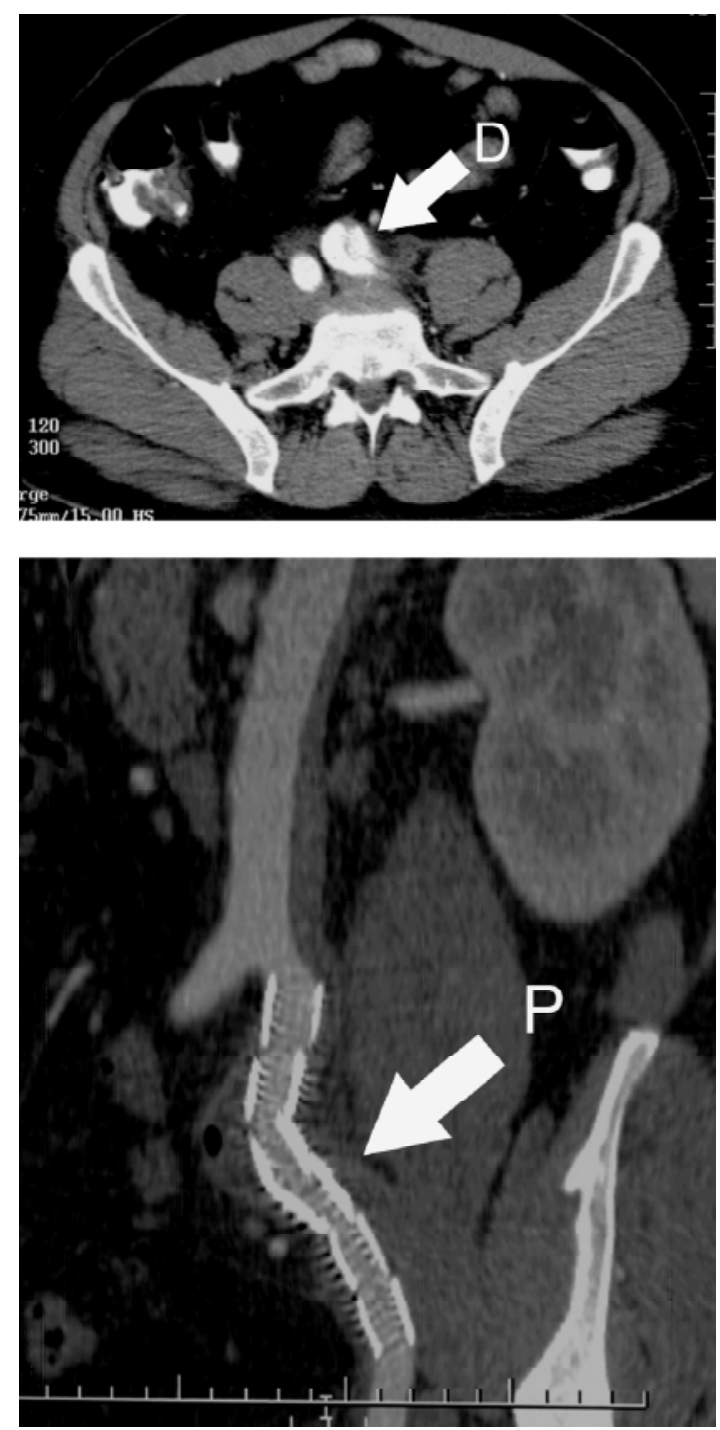

Figura 5 - T omografíaaxial computadaen corteaxial, muestra una disección aguda de la arteria iliaca común izquierda (D) con dilatación aguda del vaso al comparar con la contralateral. C ontrol con tomografíaaxial computada luego de la inserción de endoprótesis, muestra flujo a través deesta (P) con exclusión de la lesión
Parodi et al en $1991^{7}$ ha revolucionado el manejo delas enfermedades vasculares. D esdeentonces su aplicación ha sido variada, extendiéndose hacia otros territorios y permitiendo el tratamiento mínimamente invasivo de una gran variedad de patologías, particularmente de la aorta y sus ramas ${ }^{1-5,8,9}$.

Su utilización en las arterias iliacas se ha descrito y estudiado en lesiones aneurismáticas desde 199510,11, demostrando excelentes resultados a mediano y largo plazo ${ }^{12-15}$, pero su aplicación puedeser aún másamplia como se demuestra en la presente comunicación.

Las arterias iliacas son susceptibles de presentar diversas patologías, entre las que destacan los aneurismas verdaderos y pseudoaneurismas, además por su localización son susceptibles de traumatismos de diferente origen, incluyendo el iatrogénico al ser parte del acceso endovascular para múltiples procedimientosy el que puede acontecer también durante la cirugía de la columna lumbar por vía posterior.

A diferencia de la enfermedad oclusiva, habitualmente tratada con stents metálicos descubiertos, estas patologías requieren de la exclusión del segmento afectado de la circulación, esto se logra con la inserción de un dispositivo similar al stent tradicional, pero recubierto de una tela impermeable al paso de la sangre.

La utilización de esta técnica nos ha permitido resolver una variada gama de patologías en forma mínimamente invasiva, evitando grandes disecciones quirúrgicas con su consiguiente morbilidad. Por otro lado, el tratamiento quirúrgico convencional de lesionestraumáticas, ya sea ruptura o fístulas arteriovenosas, puede ser complejo desde el punto de vista quirúrgico, generando importante pérdida de sangre y eventualmente lesiones de otros órganos vecinos como el uréter 0 venas pelvianas.

$\mathrm{N}$ uestros resultados en el tratamiento endovascular de lesiones iliacas mediante endoprótesis de-

Tabla 4 - Tiempos de seguimiento

\begin{tabular}{lc}
\hline Tiempo de seguimiento en meses & $\mathbf{n}(\%)$ \\
\hline $1-6$ & $3(21,4)$ \\
$7-12$ & $1(7,1)$ \\
$13-24$ & $5(35,7)$ \\
$25-36$ & $4(28,6)$ \\
M ayor de 36 & $1(7,1)$ \\
\hline
\end{tabular}


muestran que éste es seguro, brindando un resultado durable en el tiempo para la solución de un amplio espectro de patologías.

\section{Referencias}

1. Valdés $F$, Seitz J, Fava $M$, et al. Tratamiento del aneurisma aórtico abdominal por vía endovascular: experiencia inicial. Rev M ed Chil. 1998;126:1206-15.

2. M ertens $R$, Valdés $F$, Krämer $A$, Irarrázaval $M, M$ ariné $L$, Vergara J. Aneurisma roto de aorta torácica descendente: tratamiento endovascular. Rev M ed C hil. 2001;129:1439-43.

3. M ertensR, ValdésF, Krämer A, etal. T ratamiento endovascular del aneurisma de aorta torácica descendente. Rev M ed Chil. 2003;131:617-22.

4. M ertens $R$, Valdés $F$, Krämer $A, B$ ergoeing $M$, Zalaquett $R$, Baeza C, et al. T ratamiento endovascular del trauma de aorta descendente. Rev M ed Chil. 2005;133:403-8.

5. M ertens $R, V$ aldés $F, K$ rämer $A, M$ ariné $L, V$ ergara J, $V$ aldevenito M .Pseudoaneurismatraumático detroncossupraaórticos: tratamiento endovascular. Casos clínicos. Rev M ed Chil. 2002;130:1027-32.

6. D ormandy JA, Rutherford RB. M anagement of peripheral arterial disease (PAD). TASC W orking G roup. T ransAtlantic Inter-Society Concensus (TASC). J Vasc Surg. 2000;31: S1-S296.

7. ParodiJC, PalmazJC, BaroneH D. Transfemoral intraluminal graft implantation for abdominal aortic aneurysms. Ann V asc Surg. 1991;5:491-9.

8. ParodiJC. Endovascular repair of abdominal aortic aneurysms and other arterial lesions. J Vasc Surg. 1995;21:549-55.
9. M ertens $R$, Valdés $F$, Krämer $A$. T ratamiento endovascular del aneurisma de aorta. Rev Chil Cir. 2004;56:3-11.

10. Razavi M K, D ake M D, Semba CP, N yman U R, Liddell RP. Percutaneous endoluminal placement of stent-grafts for the treatment of isolated iliac artery aneurysms. Radiology. 1995;197:801-4

11. Marin ML, Veith FJ, Lyon RT, Cynamon J, Sánchez L. Transfemoral endovascular repair of iliac artery aneurysms. Am J Surg. 1995;170:179-82.

12. Cormier F, Al Ayoubi A, Laridon D, M elki JP, Fichelle JM, Cormier JM . Endovascular treatment of iliac aneurysms with covered stents. Ann Vasc Surg. 2000;14:561-6.

13. Parsons RE, Marin M L, Veith FJ, Parsons RB, Hollier L. $M$ idterm results of en dovascular stented graftsfor thetreatment of isolated iliac artery aneurysms.J V asc Surg. 1999;30:915-21.

14. Scheinert $D$, Schroder $M$, Steinkamp H, Ludwig J, Biamino $G$. Treatment of iliac artery aneurysms by percutaneous implantation of stent grafts. Circulation. 2000;102:1/1253-8.

15. Sánchez LA, Patel AV, O hki T, et al. M idterm experience with the endovascular treatment of isolated iliac aneurysms. J V asc Surg. 1999;30:907-13.

\section{Correspondencia:}

Renato $M$ ertens $M$,

Apoquindo 3990 of 601

Santiago, Chile

Fax: (56 2) 632.6812

E-mail: rmertens@med.puc.cl

O conteúdo do J Vasc B ras está disponível em português e em inglês

no site do Jornal Vascular Brasileiro em

www.jvascbr.com.br 